\title{
Fast Calculation of Breakthrough Curves in Nonisothermal Fixed-Bed Adsorbers
}

\author{
Philip A. J. Mees \\ Albert W. Gerritsen \\ Peter J. T. Verheijen \\ Department of Chemical Engineering \\ Delft University of Technology \\ 2600 GA Delft, The Netherlands
}

Application of the fast Fourier transform, to the inversion of Laplace transforms is a recent development in the solution of equations describing chemical reactors. Hsu and Dranoff (1987) describe in detail how this method can be applied. Chen and Hsu (1987) use the fast Fourier transform for the prediction of breakthrough curves of an isothermal fixed-bed adsorber. The major strength of this approach is its speed of calculation. The purpose of the present work is to investigate the limits of the method and to show how this method can also be applied for nonisothermal adsorbers.

\section{Description of the Model}

A fixed-bed adsorption column, packed with porous spherical particles, is considered. At time zero, an adsorbable component is introduced in the column inlet flow. This introduces a step change in concentration and, possibly, in temperature. The transport phenomena in the adsorption column which are taken into account are axial dispersion, external film diffusion, pore diffusion, axial heat transfer in the solid and fluid phase, external film heat transfer, and heat transfer from fluid and solid phase to the wall. Radial gradients, the pressure drop across the bed, mass accumulation in the pores, and the heat capacity of the fluid in the pores, are neglected. Other assumptions are: concentration and temperature independence of heat capacities, densities and adsorption heat, and uniformity of the wall temperature. At the solid surface, the active component in the fluid phase is assumed to be in equilibrium with the adsorbed component in the solid phase; the involved heat effect is taken into account.

After introducing dimensionless numbers, the adsorber can

Correspondence concerning this paper should be addressed to P. J. T. Verheijen. be described by the following set of partial differential equations (a detailed derivation is given by Mees, 1988):

Mass balance in a particle:

$$
\left[\frac{\partial Q}{\partial \tau}\right]_{\eta, x}=\frac{1}{P e(m, s)}\left[\frac{\partial^{2} U_{s}}{\partial \eta^{2}}+\frac{2}{\eta} \frac{\partial U_{s}}{\partial \eta}\right]_{\tau, x}
$$

Mass balance of the fluid phase:

$$
\begin{aligned}
{\left[\frac{\partial U_{f}}{\partial x}\right]_{\tau}-\frac{1}{\operatorname{Pe}(m, a)}\left[\frac{\partial^{2} U_{f}}{\partial x^{2}}\right]_{\tau}+\left[\frac{\partial U_{f}}{\partial \tau}\right]_{x} } & \\
& +\frac{3 \cdot \mu}{\operatorname{Pe}(m, s)}\left[\frac{\partial U_{s}}{\partial \eta}\right]_{\eta=1}=0
\end{aligned}
$$

Yeat balance of the fluid phase:

$$
\begin{aligned}
& {\left[\frac{\partial \Theta_{f}}{\partial x}\right]_{\tau}-\frac{1}{P e(h, f)}\left[\frac{\partial^{2} \Theta_{f}}{\partial x^{2}}\right]_{\tau}+\frac{2 \cdot \Theta_{f} \cdot W_{f}}{\operatorname{Pe}(h, f)}} \\
& +\left[\frac{\partial \Theta_{f}}{\partial \tau}\right]_{x}-\frac{3 \cdot B i(h)}{\operatorname{Pe}(h, s)} \cdot \Phi \cdot \mu \cdot\left(\Theta_{s}-\Theta_{f}\right)=0
\end{aligned}
$$

Heat balance of the solid phase:

$$
\begin{aligned}
\frac{-1}{\operatorname{Pe}(h, s)}\left[\frac{\partial^{2} \Theta_{s}}{\partial x^{2}}\right]_{\tau}+\frac{2 \cdot \Theta_{s} \cdot W_{s}}{\operatorname{Pe}(h, s)}+\left[\frac{\partial \Theta_{s}}{\partial \tau}\right]_{x} \\
+\frac{3 \cdot B i(h)}{\operatorname{Pe}(h, s)} \cdot\left(\Theta_{s}-\Theta_{f}\right)-\frac{3 \cdot \Gamma}{\operatorname{Pe}(m, s)}\left[\frac{\partial U_{s}}{\partial \eta}\right]_{\eta=1}=0
\end{aligned}
$$

Adsorption equilibrium:

$$
Q=Q\left(U_{s}, \Theta_{s}\right)
$$


Initial and boundary conditions are given by:

$$
\begin{aligned}
& \left.\frac{\partial U_{s}}{\partial \eta}\right|_{\eta \rightarrow 0}=0 \\
& \left.\frac{\partial U_{s}}{\partial \eta}\right|_{\eta-1}=B i(m)\left[U_{f}-\left.U_{s}\right|_{\eta-1}\right] \\
& \left.Q\right|_{\text {T-0 }}=0 \\
& \left.U_{s}\right|_{T-0}=0 \\
& \left.\frac{\partial U_{f}}{\partial x}\right|_{x=0^{+}}=\operatorname{Pe}(m, a)\left[\left.U_{f}\right|_{x=0^{+}}-\left.U_{f}\right|_{x=0^{-}}\right] \\
& \left.U_{f}\right|_{x \rightarrow \infty}=0 \\
& \left.U_{f}\right|_{r=0}=0 \\
& \left.\frac{\partial \Theta_{f}}{\partial x}\right|_{x=0^{+}}=\operatorname{Pe}(h, f)\left[\left.\Theta_{f}\right|_{x=0^{+}}-\left.\Theta_{f}\right|_{x=0^{-}}\right] \\
& \left.\Theta_{f}\right|_{x \rightarrow \infty}=0 \\
& \left.\Theta_{j}\right|_{\tau-0}=0 \\
& \left.\frac{\partial \Theta_{s}}{\partial x}\right|_{x=0^{+}}=0 \\
& \left.\Theta_{s}\right|_{x \rightarrow \infty}=0 \\
& \left.\Theta_{s}\right|_{\tau \rightarrow 0}=0
\end{aligned}
$$

In the limit of the isothermal case, these equations reduce to the equations of Chen and Hsu (1987), with the exception of boundary condition, Eq. 10. Here a Danckwerts-type boundary condition is used, where Chen and Hsu use $U(x=0, \tau)=1$. In the limit of the axial dispersion coefficient equal to zero, these become identical.

The model equations are solved by Laplace transformation with respect to time $(\tau)$ and space $(x)$. To successfully apply the Laplace transform method, the equations have to be linear functions of concentrations, temperatures and their derivatives. This is valid for the present model equations, Eqs. 1-4, but not for the adsorption isotherm, Eq. 5. However, the latter can be approximated by a linear function in the pore concentration and the temperature of the solid,

$$
Q=K_{U} \cdot U_{s}+K_{\theta} \cdot \Theta_{s}
$$

In the case of an isothermal bed, the final stationary state determines $K_{U}$ (Figure 1). In the general case, the constants, $K_{U}$ and $K_{\theta}$, must be chosen such that Eq. 19 gives an approximation of the isotherm for the concentrations and temperatures between the initial and final states; and such that it reproduces these states correctly.

\section{Method of Solution}

Laplace transformation with respect to time, results in functions of $s$ and $x$. These functions are marked by a dash (-).

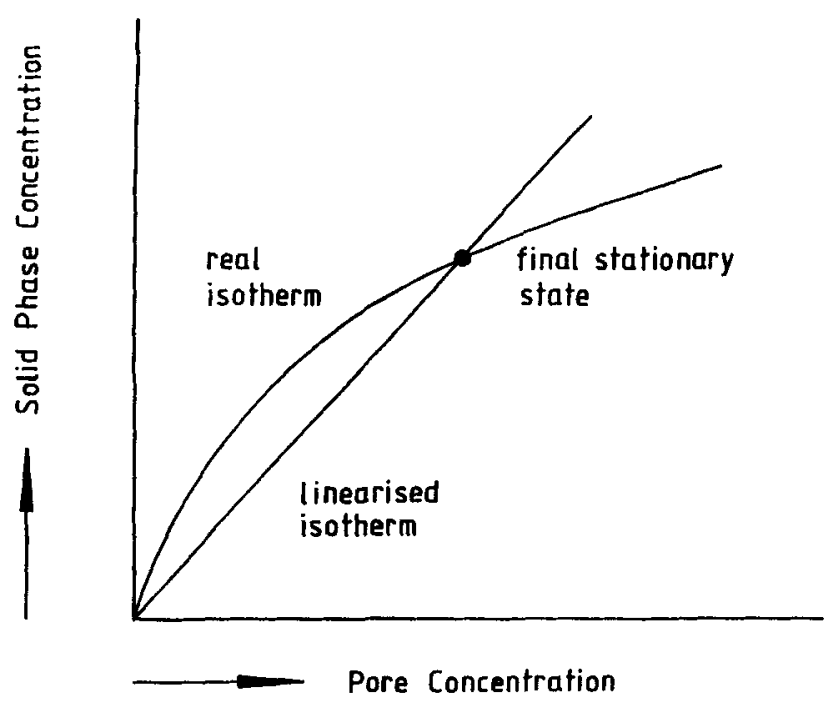

Figure 1. Linearized equilibrium curve for an isothermal bed.

Laplace transformation with respect to space, $x$, results in functions of $s$ and $y$, marked by a dash and a circumflex $\left({ }^{\wedge}\right)$.

Twice Laplace transforming the five partial differential equations, $1-4$ and 19 , results in five linear equations of $\hat{\bar{U}}_{f}, \hat{U}_{s}$, $\hat{\bar{Q}}, \hat{\boldsymbol{\theta}}_{f}$ and $\hat{\bar{\Theta}}_{s}$, three of which can be written as a matrix equation, Eq. 20, and the others given by Eqs. 21 and 22:

$$
\left[\begin{array}{ccc}
\mathrm{A} & \mathrm{B} & 0 \\
\mathrm{D} & \mathrm{E} & \mathrm{F} \\
0 & \mathrm{H} & \mathrm{I}
\end{array}\right] \cdot\left[\begin{array}{c}
\hat{\vec{U}}_{f} \\
\hat{\vec{\Theta}}_{s} \\
\dot{\vec{\Theta}}_{f}
\end{array}\right]=\left[\begin{array}{c}
R_{1} \\
R_{2} \\
R_{3}
\end{array}\right]
$$

with, for the left-hand matrix:

$$
\begin{aligned}
& A=\frac{-y^{2}}{\operatorname{Pe}(m, a)}+y+s+\frac{3 \cdot \mu \cdot \Phi_{s}}{\operatorname{Pe}(m, s)} \\
& B=\frac{3 \cdot \mu \cdot \Phi_{s}}{\operatorname{Pe}(m, s)} \cdot \frac{K_{\theta}}{K_{U}} \\
& D=-\frac{3 \cdot \Gamma \cdot \Phi_{s}}{\operatorname{Pe}(m, s)} \\
& E=\frac{-y^{2}}{P e(h, s)}+s+W_{s} \frac{2}{P e(h, s)} \\
& +\frac{3 \cdot B i(h)}{\operatorname{Pe}(h, s)}-\frac{3 \cdot \Gamma \cdot \Phi_{s}}{\operatorname{Pe}(m, s)} \cdot \frac{K_{\theta}}{K_{U}} \\
& F=-\frac{3 \cdot B i(h)}{P e(h, s)} \\
& H=-\frac{3 \cdot B i(h)}{P e(h, s)} \cdot \Phi \cdot \mu \\
& I=s+y-\frac{y^{2}}{\operatorname{Pe}(h, f)}+W_{f} \cdot \frac{2}{\operatorname{Pe}(h, f)} \\
& +\frac{3 \cdot B i(h)}{\operatorname{Pe}(h, s)} \cdot \Phi \cdot \mu
\end{aligned}
$$


And the result on the right-hand side:

$$
\begin{aligned}
& R_{1}=b_{0}-\frac{y \cdot b_{0}+b_{1}}{P e(m, a)} \\
& R_{2}=-\frac{y \cdot g_{s 0}+g_{s 1}}{P e(h, s)} \\
& R_{3}=g_{f 0}-\frac{y \cdot g_{f 0}+g_{f 1}}{P e(h, f)}
\end{aligned}
$$

The constants, $b$ and $g$, are set by the boundary conditions:

$$
\begin{array}{ll}
b_{0}=\left.\bar{U}_{f}\right|_{x=0} & b_{1}=\left.\frac{\partial \bar{U}_{f}}{\partial x}\right|_{x=0} \\
g_{f 0}=\left.\bar{\Theta}_{f}\right|_{x=0} & g_{f 1}=\left.\frac{\partial \bar{\Theta}_{f}}{\partial x}\right|_{x=0} \\
g_{s 0}=\left.\bar{\Theta}_{s}\right|_{x=0} & g_{s 1}=\left.\frac{\partial \bar{\Theta}_{s}}{\partial x}\right|_{x=0}
\end{array}
$$

The function, $\Phi_{s}$, is defined as:

$$
\begin{aligned}
& \Phi_{s}=\frac{\sqrt{ } \sigma \cdot \cos h \sqrt{ } \sigma-\sin h \sqrt{ } \sigma}{\frac{\sqrt{ } \sigma \cdot \cos h \sqrt{ } \sigma-\sin h \sqrt{ } \sigma}{B i(m)}+\sin h \sqrt{ } \sigma} \\
& \sigma=K_{U} \cdot s \cdot \operatorname{Pe}(m, s)
\end{aligned}
$$

The concentrations in the solid are directly found:

$$
\begin{gathered}
\hat{\vec{U}}_{s}=\left[\hat{\bar{U}}_{f}+\frac{K_{\theta}}{K_{U}} \cdot \hat{\bar{\Theta}}_{s}\right] \frac{\sinh (\eta \sqrt{ } \sigma)}{\eta} \\
\cdot\left[\frac{\sqrt{ } \sigma \cdot \cos h \sqrt{ } \sigma-\sin h \sqrt{ } \sigma}{B i(m)}+\sinh \sqrt{ } \sigma\right]^{-1}-\frac{K_{\theta}}{K_{U}} \cdot \hat{\bar{\Theta}}_{s} \\
\hat{\bar{Q}}=K_{U} \cdot \hat{\bar{U}}_{s}+K_{\theta} \cdot \hat{\bar{\Theta}}_{s}
\end{gathered}
$$

From Eq. 20, $\hat{\bar{U}}_{f}, \hat{\bar{\Theta}}_{f}$, and $\hat{\bar{\Theta}}_{s}$ can be solved. After that, $\hat{\bar{U}}_{s}$ and $\hat{Q}$ can be solved from Eqs. 21 and 22 .

The inverse Laplace transform with respect to $x$ is calculated by the method of residues (Churchill, 1958). The inverse Laplace transform with respect to $\tau$ must be calculated numerically. Hsu and Dranoff (1987) describe the inverse Laplace transformation by applying the fast Fourier transform. The discrete inverse Laplace transform is given by the following formula:

$f(j \Delta T)=\frac{\exp (a j \Delta T)}{2 T} \sum_{k=0}^{N-1} \cdot[F(a+i k \pi / T) \cdot \exp (i 2 \pi j k / N)]$

$$
j=0,1,2, \ldots, N-1
$$

with

$$
\Delta T=2 T / N
$$

$T$ is half the time period considered and $N$ is the number of points. $a$ is the real part of the Laplace $s$-value and determines the place where the line-integral of the inverse Laplace transform in the complex $s$-plane is calculated. The value of $a$ must be greater than the real parts of the singularities of the Laplacedomain function.

The choice of the constant, $a$, is crucial. If the value of $a$ is too large $(\gg 1 / T)$ then the first factor in Eq. 23 will amplify any numerical inaccuracies. However, if the value of $a$ is too small $(\ll 1 / T)$, then $F(a+i k \pi / T)$ is near the rightmost singularity at $s=0$, and will only vary smoothly at a high sampling density.

Chen and Hsu (1987) remove this singularity by multiplying $F(s)$ with $s$, and setting the value of $a$ to zero. This results in the derivative of the breakthrough curves, which needs to be integrated numerically, but was found unsatisfactory for a number of problems. The alternative we used is to subtract the stationary value of the time-function, divided by $s$, from the Laplace function. The constant, $a$, can now be chosen more freely (typically on the order of $1 / T$ ).

Equation 23 was calculated by applying the fast Fourier transform to $F(a+i k \pi / T)$ and multiplying the result by $\exp (a j \Delta T)$. For Eq. 23, the function in the $(s, x)$ domain must
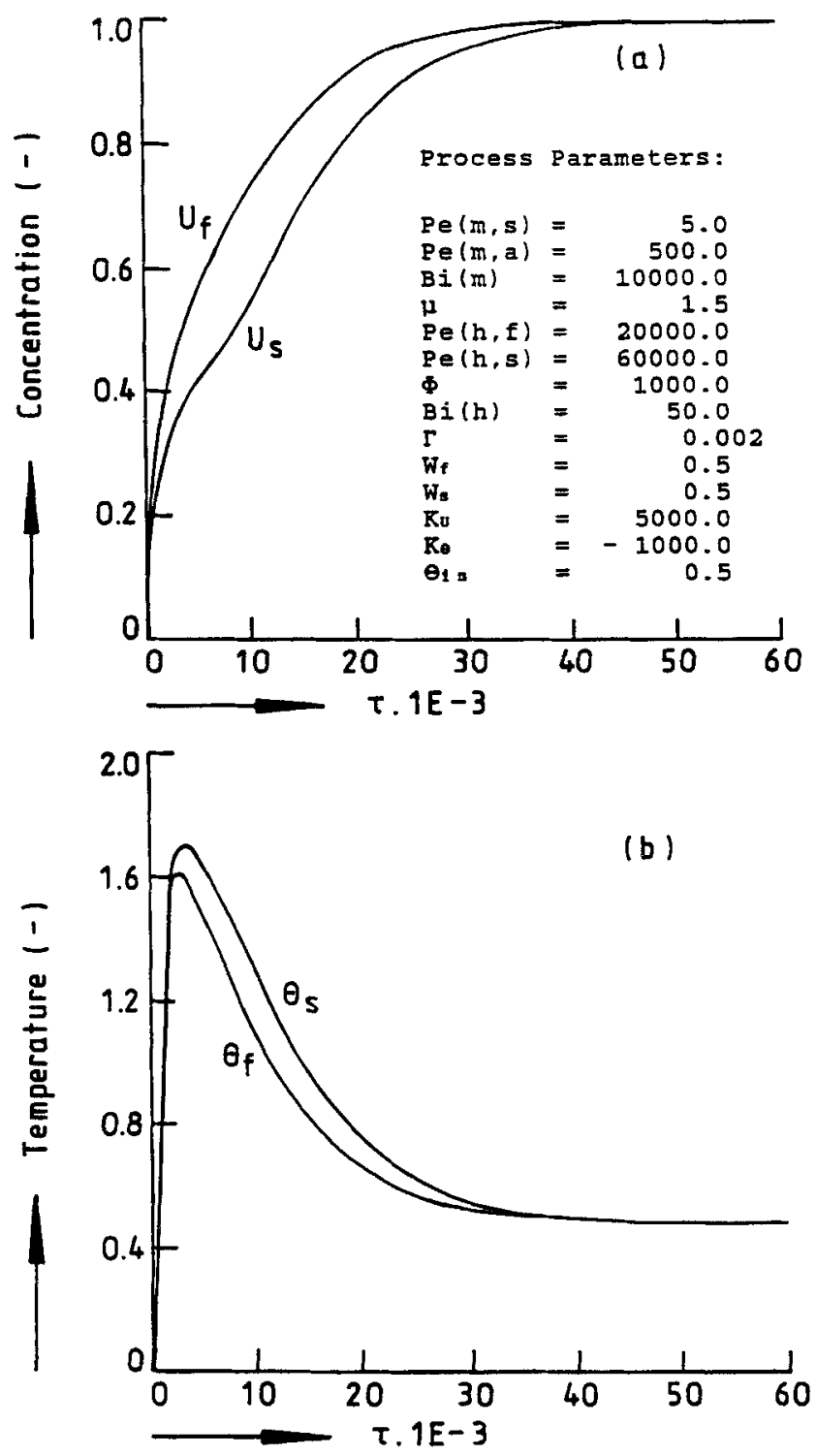

Figure 2. Example breakthrough curves. 
be calculated for a range of $s$-values, which will take a large proportion, on the order of $80 \%$, of the calculation time.

\section{Results and Discussion}

It has been verified that in the limit of the isothermal case with zero axial dispersion, the results are identical to those of Chen and Hsu (1987). Further, the family of breakthrough curves described by Pan and Basmadjian (1970) was simulated. Figure 2 shows an illustrative result of one of these; the lower fluid concentration in the center of the particles and the temperature difference between the solid and fluid phase can be seen clearly. In Table 1, a comparison of execution time (for an 8 $\mathrm{MHz}$, IBM compatible XT with mathematical coprocessor) and accuracy for the fluid concentration and temperature breakthrough curves of Figure 2 is given.

Table 1. Accuracy and Execution Time for a Fluid Concentration and Temperature Breakthrough Curve

\begin{tabular}{|c|c|c|c|c|}
\hline \multirow[b]{2}{*}{$\tau$} & \multicolumn{4}{|c|}{ Number of Sample Points } \\
\hline & $N=32$ & $N=64$ & $N=256$ & $N=1,024$ \\
\hline \multicolumn{5}{|c|}{ Fluid Concentration } \\
\hline 3,750 & 0.516176 & 0.514857 & 0.515050 & 0.515060 \\
\hline 7,500 & 0.652442 & 0.651881 & 0.651882 & 0.651887 \\
\hline 11,250 & 0.771038 & 0.771002 & 0.771072 & 0.771080 \\
\hline 15,000 & 0.858078 & 0.858144 & 0.858214 & 0.858221 \\
\hline 18,750 & 0.915872 & 0.915909 & 0.915956 & 0.915961 \\
\hline 22,500 & 0.951814 & 0.951789 & 0.951810 & 0.951813 \\
\hline 26,250 & 0.973163 & 0.973072 & 0.973069 & 0.973070 \\
\hline 30,000 & 0.985435 & 0.985276 & 0.985251 & 0.985250 \\
\hline 33,750 & 0.992341 & 0.992101 & 0.992051 & 0.992050 \\
\hline 37,500 & 0.996203 & 0.995853 & 0.995772 & 0.995769 \\
\hline 41,250 & 0.998402 & 0.997899 & 0.997777 & 0.997772 \\
\hline 45,000 & 0.999732 & 0.999018 & 0.998844 & 0.998836 \\
\hline 48,750 & 1.000658 & 0.999642 & 0.999405 & 0.999397 \\
\hline 52,500 & 1.001688 & 1.000036 & 0.999695 & 0.999689 \\
\hline 56,250 & 1.008600 & 1.001227 & 0.999853 & 0.999840 \\
\hline \multicolumn{5}{|c|}{ Fluid Temperature } \\
\hline 3,750 & 1.589755 & 1.581132 & 1.580621 & 1.580710 \\
\hline 7,500 & 1.298788 & 1.294765 & 1.293977 & 1.293968 \\
\hline 11,250 & 1.030666 & 1.028211 & 1.027626 & 1.027610 \\
\hline 15,000 & 0.830449 & 0.828711 & 0.828266 & 0.828251 \\
\hline 18,750 & 0.694835 & 0.693583 & 0.693256 & 0.693245 \\
\hline 22,500 & 0.608779 & 0.607879 & 0.607645 & 0.607638 \\
\hline 26,250 & 0.556651 & 0.556003 & 0.555840 & 0.555837 \\
\hline 30,000 & 0.526133 & 0.525673 & 0.525564 & 0.525564 \\
\hline 33,750 & 0.508704 & 0.508397 & 0.508335 & 0.508338 \\
\hline 37,500 & 0.498923 & 0.498751 & 0.498732 & 0.498739 \\
\hline 41,250 & 0.493515 & 0.493450 & 0.493467 & 0.493478 \\
\hline 45,000 & 0.490677 & 0.490600 & 0.490617 & 0.490632 \\
\hline 48,750 & 0.489693 & 0.489200 & 0.489094 & 0.489111 \\
\hline 52,500 & 0.491204 & 0.488964 & 0.488297 & 0.488304 \\
\hline 56,250 & 0.508621 & 0.492849 & 0.487989 & 0.487887 \\
\hline Execution Time & $18.90 \mathrm{~s}$ & $34.30 \mathrm{~s}$ & $128.60 \mathrm{~s}$ & $506.70 \mathrm{~s}$ \\
\hline \multicolumn{5}{|c|}{ Process Parameters } \\
\hline \multicolumn{2}{|c|}{$P e(m, s)=5.0$} & \multicolumn{3}{|c|}{$B i(h)=50.0$} \\
\hline \multicolumn{2}{|c|}{$\operatorname{Pe}(m, a)=500.0$} & \multicolumn{3}{|c|}{$\Gamma=0.002$} \\
\hline \multicolumn{2}{|c|}{$B i(m)=10,000.0$} & \multicolumn{3}{|c|}{$W_{f}=0.5$} \\
\hline \multirow{2}{*}{\multicolumn{2}{|c|}{$\begin{aligned} \mu & =1.5 \\
P e(h, f) & =20,000.0\end{aligned}$}} & \multicolumn{3}{|c|}{$W_{s}=0.5$} \\
\hline & & \multicolumn{3}{|c|}{$K u=5,000.0$} \\
\hline \multirow{2}{*}{\multicolumn{2}{|c|}{$\begin{aligned} P e(h, s) & =60,000.0 \\
\Phi & =1,000.0\end{aligned}$}} & \multicolumn{3}{|c|}{$K \theta=-1,000.0$} \\
\hline & & \multicolumn{3}{|c|}{$\Theta_{i n}=0.5$} \\
\hline
\end{tabular}

The speed of this technique could not be compared with other methods, because the data was not available. Chen and $\mathrm{Hsu}$ (1987) however, compared the speed of their calculation for isothermal adsorption processes with earlier methods, and found that their method was faster by more than three orders of magnitude. The isothermal problem that Chen and Hsu (1987) solved in $0.3 \mathrm{~s}$ on a CDC Cyber 850 , took $20 \mathrm{~s}$ on the above described PC. A nonisothermal case with an equal number of points would take, respectively, $5 \mathrm{~s}$ (by estimation) and $500 \mathrm{~s}$ (Table 1), which is two orders of magnitude faster than earlier methods for the isothermal case. Because of this fast calculation, the method can be used satisfactorily in parameter estimation routines (Mees et al., 1989).

Chen and Hsu (1989) have recently considered the linearity of the isotherm, Eq. 19, which is the limitation of this method. They provide an iterative solution for the special case of a Langmuir kinetics controlled process. It remains to be proven that general kinetics combined with heat effects can be solved without losing the elegance and speed of this method.

\section{Notation}

$c_{f}, c_{s}=$ compound concentration in fluid and pores $/ \mathrm{m}^{3}$ fluid, respectively, $\mathrm{mol} / \mathrm{m}^{3}$

$c_{0}=$ incoming fuid concentration, $\mathrm{mol} / \mathrm{m}^{3}$

$C p_{f}, C p_{s}=$ heat capacity of fluid and solid, respectively, $J / \mathrm{kg} \cdot \mathrm{K}$

$D_{a}=$ axial mass dispersion coefficient of fluid, $\mathrm{m}^{2} / \mathrm{s}$

$D_{p}=$ diffusion coefficient in pores, $\mathrm{m}^{2} / \mathrm{s}$

$h_{f}, h_{w f}, h_{w s}=$ heat transfer coefficient, film, fluid to wall, and solid to wall, respectively, $J /\left(\mathrm{m}^{2} \cdot \mathrm{s} \cdot \mathrm{K}\right)$

$-\Delta H=$ adsorption heat, $\mathbf{J} / \mathrm{mol}$

$k_{f}=$ film mass transfer coefficient, $\mathrm{m} / \mathrm{s}$

$L=$ reactor length, $\mathrm{m}$

$q=$ adsorbed concentration per $\mathrm{m}^{3}$ solid, $\mathrm{mol} / \mathrm{m}^{3}$

$R, R_{p}=$ reactor and particle radius, respectively, $\mathrm{m}$

$r_{p}=$ radial place in particle, $m$

$t=$ time, s

$T_{f}=$ fluid temperature, $\mathrm{K}$

$T_{i n}, T_{s}, T_{w}=$ temperature of incoming fluid, solid, and wall, respectively, $\mathrm{K}$

$v=$ intrinsic fluid velocity, $\mathrm{m} / \mathrm{s}$

$z$ = axial place in reactor, $\mathrm{m}$

\section{Greek letters}

$\epsilon=$ bed porosity, -

$\lambda_{f a}=$ axial heat dispersion coefficient of fluid, $\mathrm{J} /(\mathrm{m} \cdot \mathrm{s} \cdot \mathrm{K})$

$\lambda_{s a}=$ axial heat conduction coefficient of solid, $J /(\mathrm{m} \cdot \mathrm{s} \cdot \mathrm{K})$

$\rho_{f}=$ fluid density, $\mathrm{kg} / \mathrm{m}^{3}$

$\rho_{s}=$ solid density per $\mathrm{m}^{3}$ particles, $\mathrm{kg} / \mathrm{m}^{3}$

\section{Dimensionless parameters}

$$
\begin{aligned}
\tau & =t \cdot v / L \\
\alpha & =L / R \\
\beta & =R / R_{p} \\
x & =z / L \\
\eta & =r_{p} / R_{p} \\
U_{f} & =c_{f} / c_{0} \\
U_{s} & =c_{s} / c_{0} \\
Q & =q / c_{0} \\
\Theta_{f} & =\left(T_{f}-T_{w}\right) / T_{w} \\
\Theta_{s} & =\left(T_{s}-T_{w}\right) / T_{w} \\
\Theta_{i n} & =\left(T_{i n}-T_{w}\right) / T_{w} \\
\operatorname{Pe}(m, a) & =L \cdot v / D_{a} \\
P e(m, s) & =R_{p}^{2} \cdot v /\left(L \cdot D_{p}\right) \\
P e(h, f) & =L \cdot v \cdot \rho_{f} \cdot C p_{f} / \lambda_{f a} \\
\operatorname{Pe}(h, s) & =L \cdot v \cdot \rho_{s} \cdot C p_{s} / \lambda_{s a} \\
B i(m) & =k_{f} \cdot R_{p} / D_{p} \\
B i(h) & =h_{f} \cdot L^{2} /\left(R_{p} \cdot \lambda_{s a}\right) \\
W_{f} & =h_{w f} \cdot L^{2} /\left(\lambda_{f a} \cdot R\right)
\end{aligned}
$$




$$
\begin{aligned}
W_{s} & =h_{w s} \cdot L^{2} /\left(\lambda_{s a} \cdot R\right) \\
\Gamma & =-\Delta H \cdot c_{0} /\left(\rho_{s} \cdot C p_{s} \cdot T_{w}\right) \\
\Phi & =p_{s} \cdot C p_{s} /\left(\rho_{f} \cdot C p_{f}\right) \\
\mu & =(1-\epsilon) / \epsilon
\end{aligned}
$$

\section{Literature Cited}

Chen, T. L., and J. T. Hsu, "Prediction of Breakthrough Curves by the Application of Fast Fourier Transform," AIChE J., 33(8), 1387 (Aug., 1987).

Chen, T. L., and J. T. Hsu, "Application of Fast Fourier Transform to Nonlinear Fixed-Bed Adsorption Problems," AIChE J., 35(2), 332 (Feb., 1989).

Churchill, R. V., Operational Mathematics, McGraw-Hill, New York, (1958).

Hsu, J. T., and J. S. Dranoff, "Numerical Inversion of Certain Laplace
Transforms by the Direct Application of Fast Fourier Transform (FFT) Algorithm," Comput. Chem. Eng., 11, 101 (1987).

Mees, Ph. A. J., "A Fast Method for the Prediction of Breakthrough Curves of Non-Isothermal Fixed-Bed Reactors," Master's Thesis, Delft University of Technology, (1988).

Mees, Ph. A. J., E. H. P. Wolf, P. J. T. Verheijen, and C. M. van den Bleek, "Fast Simulation of Non-steady State Emission Problems in Energy Conversion," International Forum on Mathematical Modelling and Computer Simulation of Processes in Energy Systems, Sarajevo, Yugoslavia (Mar., 1989).

Pan, C. Y., and D. Basmadjian, "An Analysis of Adiabatic Sorption of Single Solutes in Fixed Beds: Pure Thermal Wave Formation and Its Practical Implications," Chem. Eng. Sci., 25, 1653 (1970).

Manuscript received Oct. 12,1988, and revision received Mar. 8,1989 